\title{
construckión y posibilidades de utilizakión del aparato automático de determinación de carbonatos, sistema Holderbank
}

La industria del cemento pretende - cada vez más- llegar a la automación del proceso de fabricación, ya que se exige mejor calidad al producto final y, además, escasea la mano de obra calificada.

Pero un control automático de fabricación sólo es racional cuando puede verificarse y medirse continuamente. En la industria del cemento esta regulación tiene por objeto mantener - dentro de lo posible - una composición constante de la mezcla de materias primas, requisito ineludible para un funcionamiento uniforme de los hornos y para una calidad constante del producto final.

En primer lugar, el técnico debe decidir si es necesario para la fabricación analizar totalmente la mezcla de materias primas o si es suficiente con una determinación de carbonatos. Mientras el título sea significativo, basta generalmente un análisis parcial, es decir, la determinación del contenido en $\mathrm{CaCO}_{3}$.

El problema de la valoración automática de los polvos crudos se ha resuelto por colaboración entre el centro de Holderbank (Suiza) y la empresa Bran \& LÜBBE, de Hamburgo. El aparato automático descrito a continuación, sistema Holderbank, se ha ensayado minuciosamente en el laboratorio durante largo tiempo, bajo las más duras condiciones de trabajo y se ha acreditado por sus excelentes cualidades.

El sentido y la finalidad de este trabajo es dar una visión general sobre la construcción, funcionamiento y posibilidades de aplicación de dicho aparato Holderbank, para facilitar un juicio sobre su utilización.

\section{CONSTRUCCION Y FORMA DE FUNCIONAMIENTO}

El Holderbank es un aparato totalmente automático para volumetrías de carbonatos, construido para el control continuo del título de las pastas y polvos crudos en las fábricas de cemento. Se trata de una ingeniosa combinación de elementos ya conocidos y acredi- 
tados, que se ha adaptado a las necesidades específicas de la industria del cemento.

La instalación consta de las siguientes partes:

1) toma de muestras, diseñado tanto para fabricación por vía húmeda como para vía seca;

2) molino de martillos, que prepara el polvo crudo;

3) aparato automático de pesada, que dosifica la muestra;

4) aparato de valoración, que determina el contenido en $\mathrm{CaCO}_{3}, \mathrm{y}$

5) cuadro de mando, que determina el proceso y que puede ser acomodado, según el caso, a las condiciones de trabajo y exigencias correspondientes.

Un ciclo de trabajo de toda la instalación necesita 6 minutos, es decir, que se realizan diez volumetrías por hora. Las operaciones transcurren esquemáticamente como sigue:

La instalación de preparación suministra al aparato automático de pesada una muestra de polvo crudo, que pesa $1,250 \mathrm{~g}$, y se lleva al recipiente de reacción. El resto de la muestra se separa. En el recipiente de reacción, el crudo se descompone con $\mathrm{HCl}$ en frío. Se diluye con agua y se hace la valoración en retroceso con $\mathrm{NaOH}$ hasta un determinado punto de viraje ( $\mathrm{pH} 5,5)$. Por el $\mathrm{NaOH}$ consumido se determina automáticamente el contenido de $\mathrm{CaCO}_{3}$ en $\%$ y se comunica en forma de impulsos de corriente continua de 0 a $40 \mu \mathrm{A}$

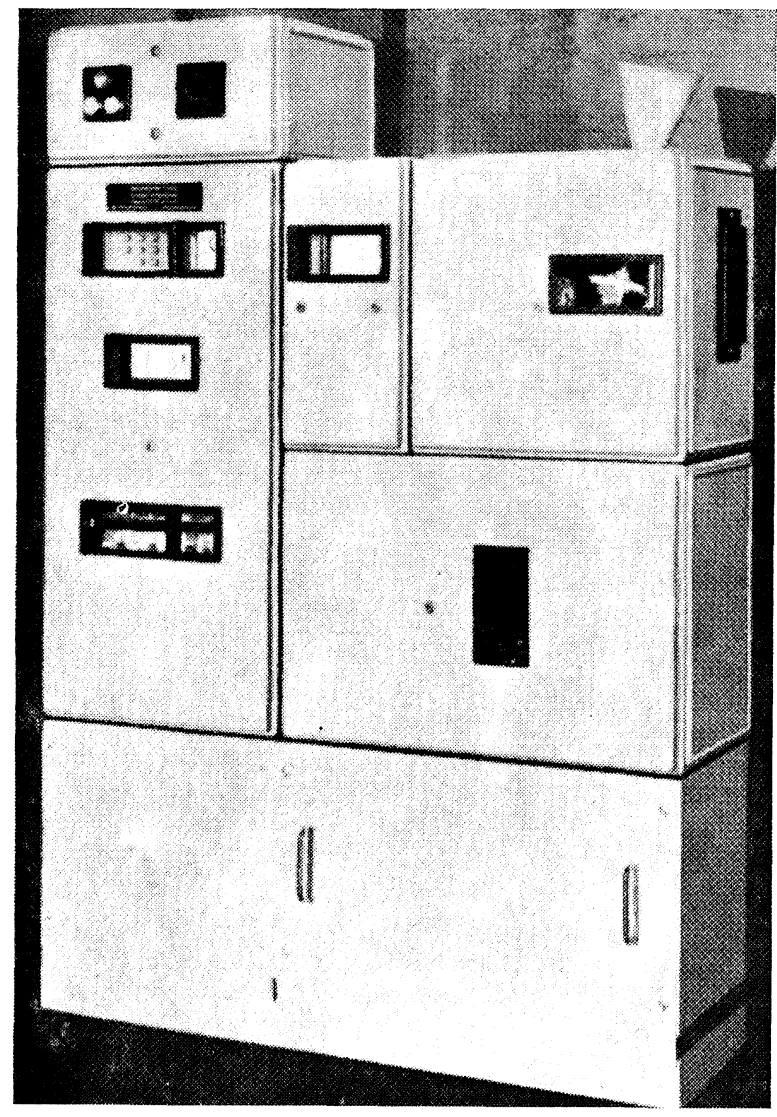

Fig. 1.-Aparato automático de valoración, sistema Holderbank, caja cerrada. a los instrumentos correspondientes (1.000 ohmios de resistencia interna). Por las variaciones en el título puede regularse la mezcla.

La aptitud de acomodación del aparato automático a las distintas condiciones de funcionamiento se basa en su construcción (figura 1).

El analizador, el grupo de mando y de programación, el sistema electrónico de balanzas y las balanzas están alojados en carcasas normalizadas. Las carcasas, cerradas con tapas de chapa de acero impermeable al polvo y al agua (tipo de protección P54), pueden cerrarse separadamente y se acomodan a las duras condiciones de trabajo de una fábrica de cemento. Las carcasas normalizadas están montadas en un conjunto sobre un bastidor macizo y sin vibraciones por disponer de amortiguadores de oscilaciones.

Esta unidad por elementos tiene las siguientes dimensiones:

A ncho: $1.250 \mathrm{~mm}$; profundidad: $325 \mathrm{~mm}$; altura: 1.750 milímetros. 


\section{DESCRIPCION DETALLADA}

\section{Tomamuestras de pasta cruda con secadero para fábricas de vía húmeda}

Para la utilización del aparato Holderbank, en fábricas de cemento que trabajan por el procedimiento por vía húmeda, se ha desarrollado un tomamuestras de pasta cruda con secadero, que prepara automáticamente, cada 6 minutos, una muestra de pasta seca de unos 50 gramos.

La figura 2 muestra el montaje de esta instalación, que funciona como a continuación se indica:

Una corriente continua lateral derivada de la corriente principal de pasta fluye a través de la esclusa del tomamuestras, en la que se abre brevemente, cada 6 minutos, una válvula accionada neumáticamente y deja pasar una muestra de pasta a la cinta de secado. Un rastrillo vibrante distribuye uniformemente la muestra sobre la cinta de acero, que pasa lentamente a través del horno túnel. Este horno está calentado eléctricamente. Las calefacciones inferior y superior pueden regularse independientemente entre sí y sin escalonamientos. Sobre la polea de la cinta situada a la salida del horno se divide la torta de polvo crudo seca mediante ruedas cortantes y se rasca entonces de la cinta.

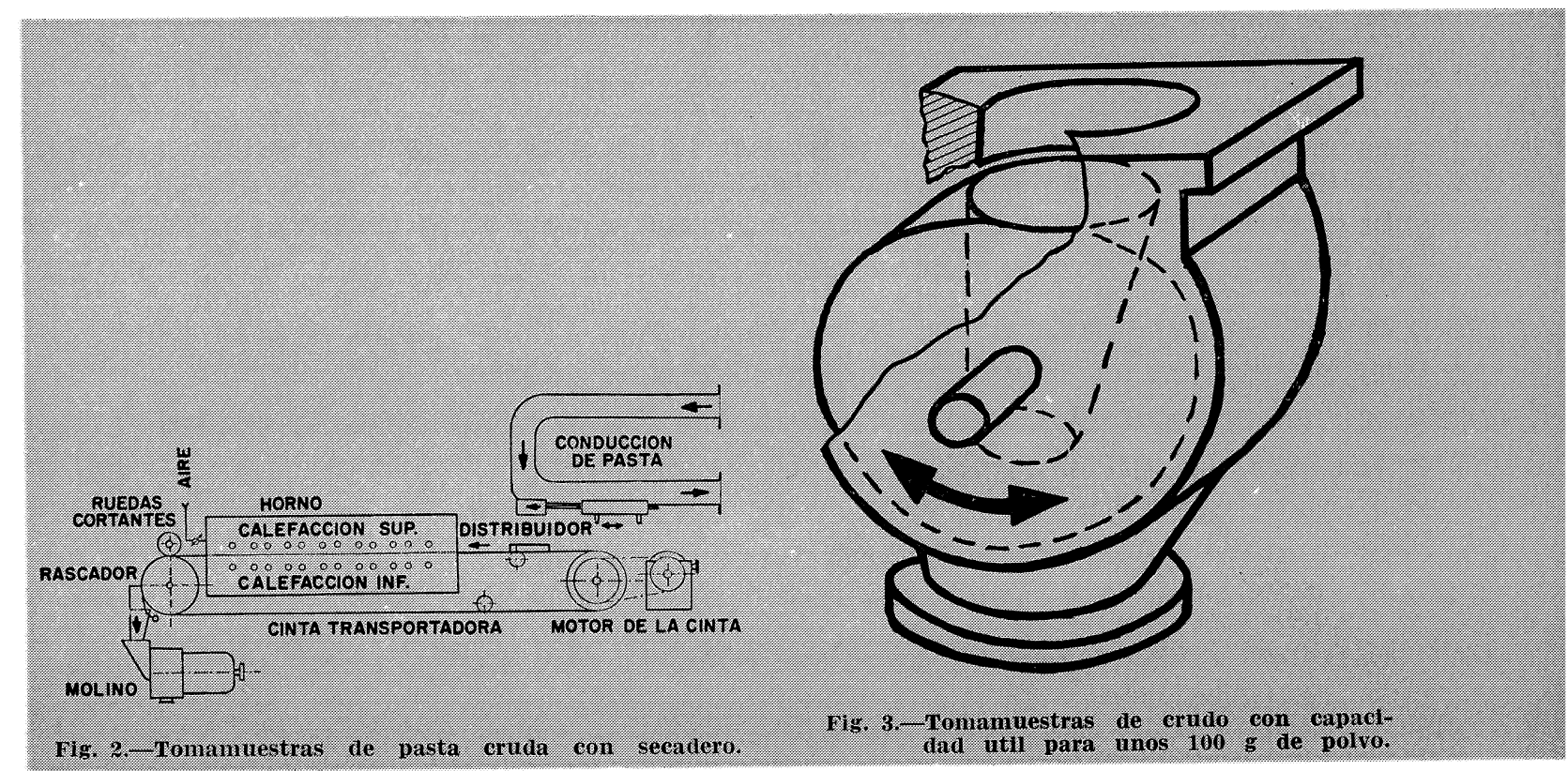

El molino de martillos dispuesto bajo la polea desmenuza la muestra. En la tolva situada bajo el molino se va acumulando el producto molido, que va a parar directamente a los aparatos automáticos de dosificación de pesada.

Esta instalación recibe la corriente eléctrica y los impulsos de mando desde una unidad situada en el aparato de valoración automático.

La preparación de una muestra de pasta dura 6 minutos. Las dimensiones de la instalación son:

Longitud: $1.700 \mathrm{~mm}$; ancho: $500 \mathrm{~mm}$; altura: 1.000 milímetros. 


\section{Tomamuestras de polvo crudo}

Para las fábricas que trabajan por vía seca puede utilizarse un tomamuestras corriente, tal como el que ya se ha acreditado en varias fábricas.

Esencialmente se ha de tener en cuenta que los dispositivos automáticos de dosificación de pesada han de alimentarse cada 6 minutos con una muestra representativa de unos $50 \mathrm{~g}$ de polvo crudo.

Para esta instalación se utiliza el tomamuestras de crudo descrito a continuación (fig. 3), que ha sido desarrollado y probado en colaboración con el Centro Técnico de Holderbank:

De la conducción del crudo se extrae continuamente una pequeña muestra de polvo mediante un tornillo sin fin, una ranura o una cinta transportadora y se conduce hasta el tomamuestras. Cada 6 minutos, por vuelco de la tolva colectora, se añade una muestra de 50 a 100 g al aparato automático de dosificación de pesada.

La tolva colectora está apoyada por ambos lados en dos rodamientos de bolas y se vuelıa por una rueda dentada y una cremallera accionadà por un cilindro automático.

Este dispositivo proporciona un funcionamiento impecable bajo las condiciones más severas.

Todas las piezas que giran son accesibles desde el exterior, y las empaquetaduras se realizan con anillos labiales o toroidales. Por lo tanto, también aquí está garantizado un cierre hermético. En la construcción se tuvo especialmente en cuenta que no existieran zonas muertas, con objeto de evitar el retardo de las muestras de polvo crudo. Este tomamuestras - como se ha mencionado- está montado directamente debajo del tornillo sin fin, o detrás de unas ranuras o cinta transportadora. En el último caso, la acumulación del producto a medir se efectúa en una tolva oscilante.

En la toma de muestras en vía seca, la construcción definitiva de los accesorios para el tomamuestras se acomoda a las condiciones locales de la fábrica de cemento.

\section{Aparato automático de dosificación de pesada Mettler}

Como se requiere gran exactitud en la valoración, fue necesario encontrar una balanza muy exacta para pesar la muestra de crudo, que debe ser de 1,250 gramos.

El aparato automático de dosificación de pesada Mettler (figura 4), descrito a continuación, satisface plenamente estos requisitos. Una muestra que no se encuentre dentro de los límites de tolerancia, es expulsada automáticamente por la balanza. En este caso, ésta vuelve a efectuar una nueva pesada automática. Después de la pesada correcta, el material sobrante se expulsa automáticamente.

Un sistema electrónico incorporado permite pesar y analizar muestras separadamente o bien realizar varias pesadas de una muestra, para hacer las mismas valoraciones de crudo. En el último caso, la duración de un ciclo de trabajo viene determinado por el número de pesadas.

El aparato automático de dosificación de pesada está provisto de una señal de alarma óptica, que responde en caso de averías (tolva de alimentación vacía, pesadas rechazadas permanentemente, etc.). Adicionalmente también puede conectarse una instalación de alarma acústica. 


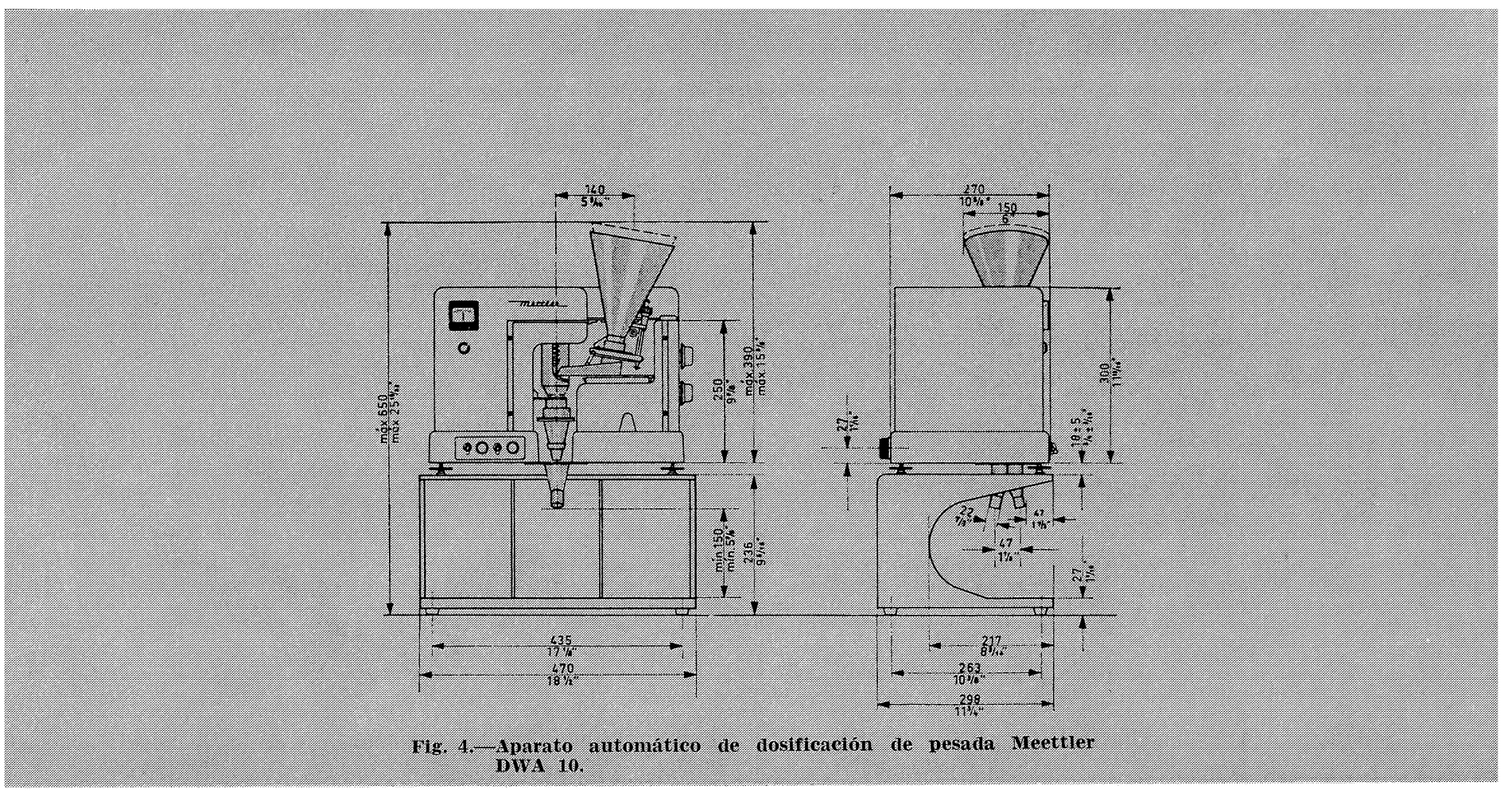

\section{Aparato de valoración Bran \& Lübbe}

En la parte inferior del aparato B y L (figura 1) está colocado el propio analizador. Este comprende el recipiente de reacción con dispositivo de alimentación de muestras y agitador, las dos bombas dosificadoras Titrodo para $\mathrm{HCl}$ y $\mathrm{NaOH}$, así como la válvula magnética para agua de lavado y de dilución.

En la parte izquierda de la unidad por elementos se hallan: las instalaciones electrónicas de la balanza Mettler y los aparatos de mando con el mecanismo conmutador de programas y el instrumento indicador. Además también se encuentran aquí montados: el relé de medida para la regulación del punto final de la titración y los potenciómetros de las bombas dosificadoras de $\mathrm{HCl}$ y $\mathrm{NaOH}$ para la regulación del factor.

El trabajo de los elementos citados se deduce del esquema de marcha (figura 5), que se describe a continuación:

Un tubo de vidrio vibrante transporta el crudo pesado desde la balanza Mettler hasta el recipiente de reacción (figura 6). De la botella de ácido colocada en el soporte se pasa una determinada cantidad de $\mathrm{HCl} 2 \mathrm{~N}$ por la bomba hasta el recipiente de reacción. Simultáneamente se ajusta en la conmutación de los programas el mecanismo de agitación. Después se empieza el proceso de disolución del crudo, que dura algo menos de 1 minuto. Durante este tiempo se añade a través de la válvula agua descarbonatada ( $50 \mathrm{ml})$ para la dilución. Una $2 .{ }^{a}$ bomba añade $\mathrm{NaOH} 0,8 \mathrm{~N}$ hasta que se alcanza el punto de viraje; $\mathrm{pH}$ 5,5 . En nuestro caso se ha elegido un $\mathrm{pH}$ de 5,5 para no valorar simultáneamente el $\mathrm{CO}_{2}$ desprendido. $\mathrm{El}$ consumo de $\mathrm{NaOH} 0,8 \mathrm{~N}$ es proporcional al porcentaje de $\mathrm{CaCO}_{3}$ existente.

Mediante el sistema electrónico el camino recorrido por la bomba Titrodos se transforma en cantidad de carbonato cálcico, se indica mediante el instrumento correspondiente y se registra continuamente en la cinta del registrador.

Durante la valoración con $\mathrm{NaOH}$, la bomba de $\mathrm{ClH}$ se acciona en sentido inverso por medio del mecanismo de conmutación de programas, es decir, que el pistón se mueve al re- 
vés y llena el recipiente esférico para la próxima valoración. Lo mismo puede decirse para la marcha de la bomba de $\mathrm{NaOH}$. Después del proceso de valoración se vacía el recipiente de reacción y se limpia lavándolo varias veces con agua. Realizada esta operación queda en disposición de efectuar un nuevo análisis.

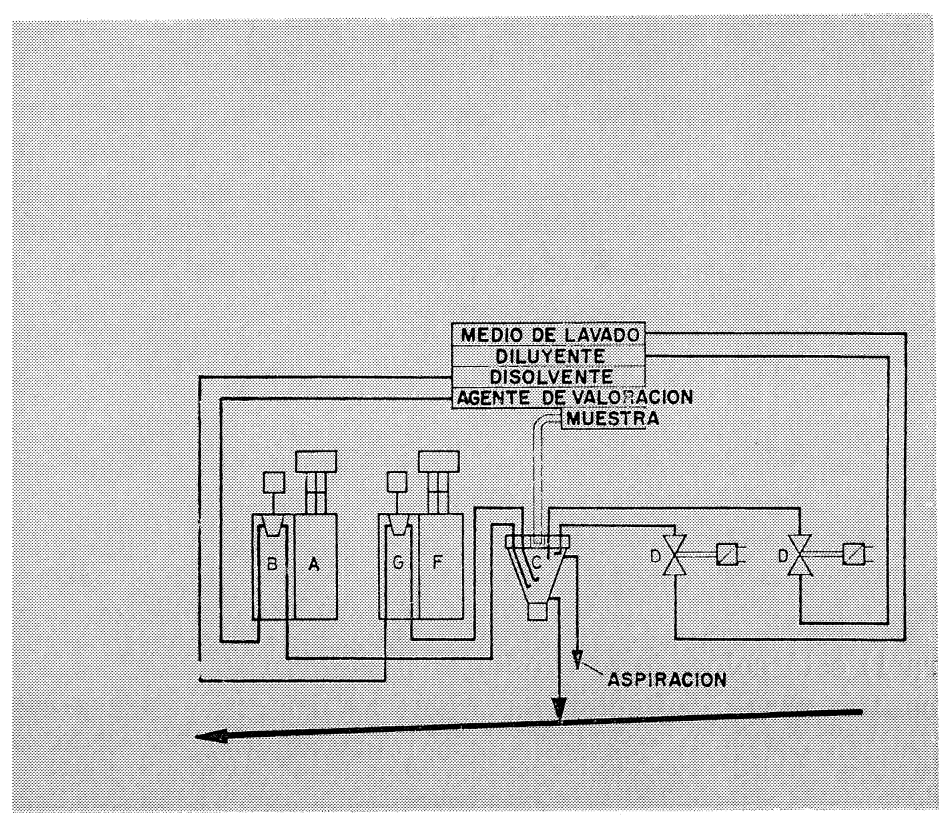

Fig. 5.-Esquema de flujos.

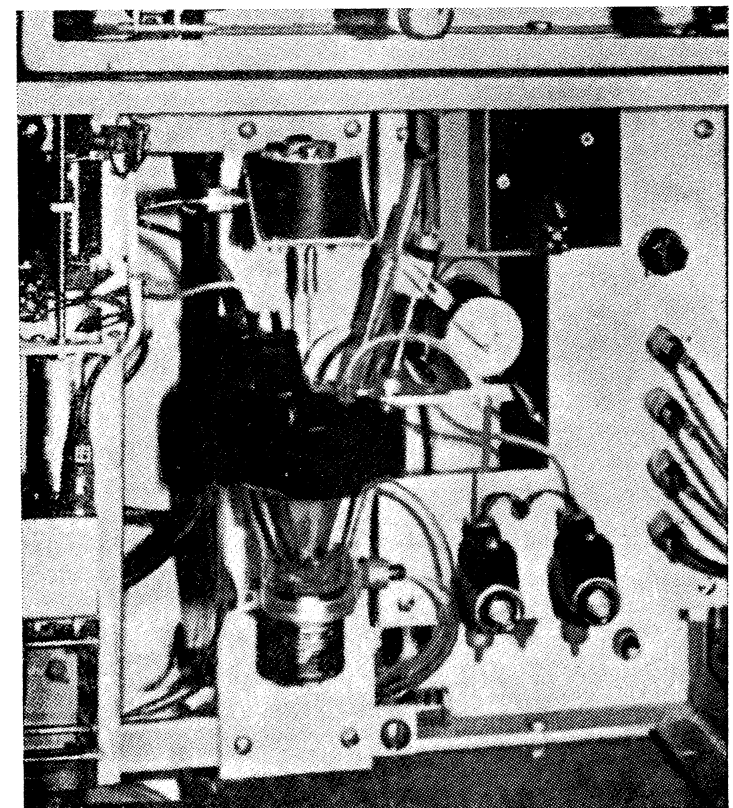

Fig. 6.-Adición de la muestra al recipiente de reacción.

\section{REQUISITOS}

\section{Polvo crudo}

Para la determinación automática del contenido de $\mathrm{CaCO}_{3}$ mediante un titrometro automático puede emplearse directamente el polvo crudo de las fábricas de vía seca, es decir, que no precisa ninguna nueva molienda.

Como dato orientativo diremos que el polvo crudo puede presentar un residuo de un $15 \%$ en 4.900 MS y de un $2 \%$ en 900 MS. Según la mineralogía y la facilidad de reacción, también puede tolerarse polvos crudos con un residuo de un $25 \%$ en 4.900 MS.

\section{Condiciones generales}

Para el funcionamiente del aparato automático Holderbank se necesita no sólo agua natural para el lavado del recipiente de reacción, sino también agua descarbonatada o ablandada para la dilución.

Además se precisa, para la conexión eléctrica, corriente alterna 220/380 V, $50 \mathrm{~Hz}$.

Para el accionamiento neumático de algunas partes del tomamuestras de pasta cruda ha de disponerse de aire comprimido a una presión de $1,2 \mathrm{kp} / \mathrm{cm}^{2}$ o más. 


\section{CONSERVACION DEL APARATO}

Además de dos revisiones anuales completas del aparato, por personal especializado de Bran und LÜBBE de Hamburgo, se ha de contar con los siguientes servicios preventivos:

- diariamente: 1/2 hora para la inspección general, así como para comprobar el registrador, los recipientes de reactivos y el funcionamiento general de toda la valoración;

- semanalmente: de 3 a 4 horas para limpieza de la alimentación del polvo crudo o cemento, de la balanza, del recipiente de reacción y para el relleno de reactivos, etc. No es preciso otro mantenimiento.

\section{DATOS TECNICOS}

El campo normal de medida del aparato automático de titración oscila de 60 a $100 \%$ de $\mathrm{CaCO}_{3}$. Naturalmente, también son posibles otros campos de medida según la composición del crudo.

El error del aparato es, en el citado campo de medida, de $\pm 0,4 \%$ de $\mathrm{CaCO}_{3}$. La desviación standard de los valores medidos con el mismo polvo crudo, calculada como valor de la media aritmética, se encuentra entre $\pm 0,12$ y $\pm 0,16$.

Con cada aparato automático se suministra la correspondiente hoja de características (figura 7) y las instrucciones detalladas del funcionamiento.

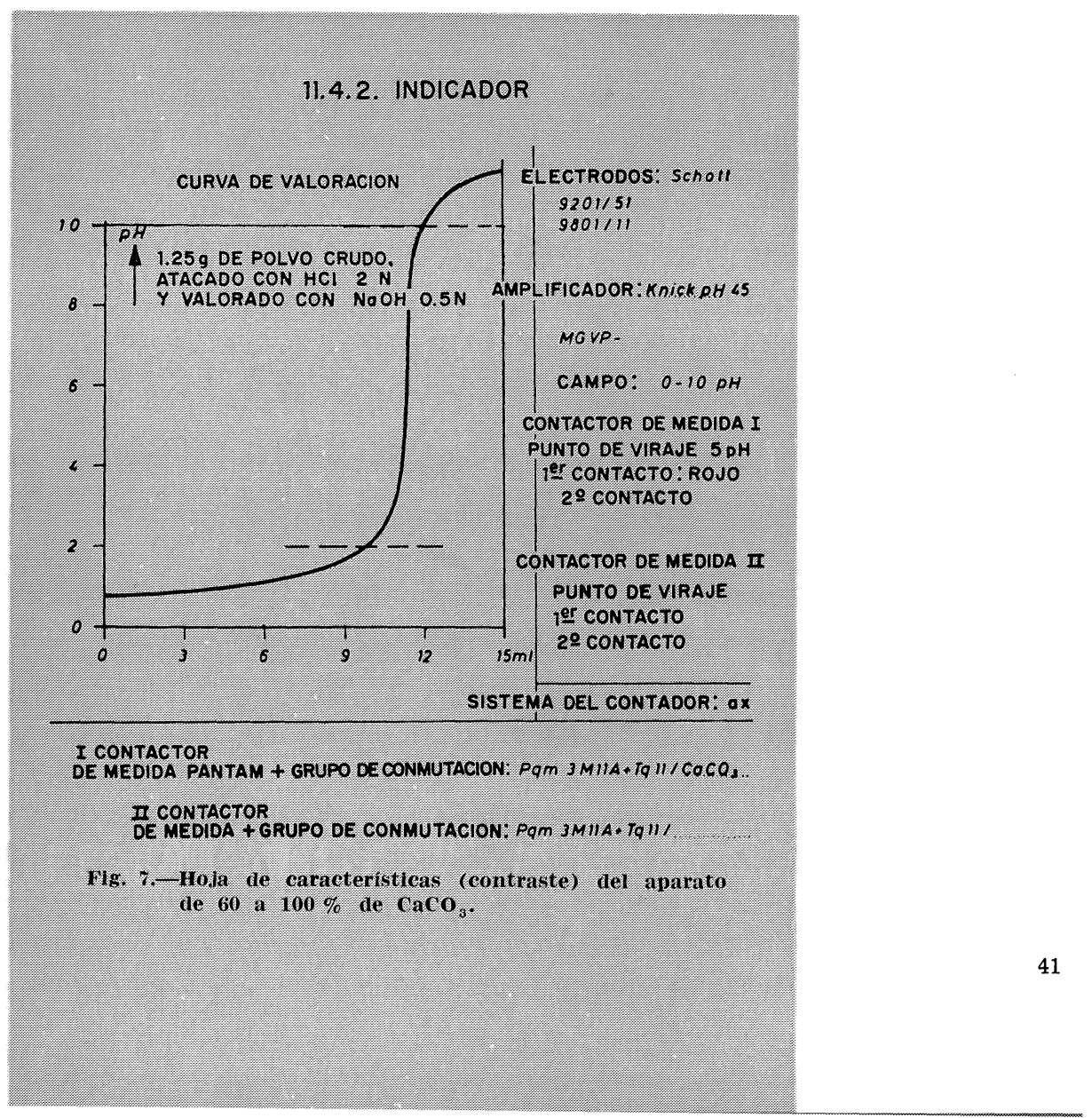

